\title{
ENTREVISTA COM ALVARO ECHEVERRI
}

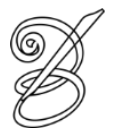 \\ Patrícia Rodrigues $\operatorname{COSTA}^{1,2}$ \\ Doutoranda em Estudos da Tradução \\ Programa de Pós-Graduação em Estudos da Tradução (PGET) \\ Universidade Federal de Santa Catarina (UFSC) \\ prcosta1986@gmail.com \\ Germana Henriques Pereira de SOUSA ${ }^{3}$ \\ Doutora em Literatura \\ Professora na Universidade de Brasília (UnB) \\ Programa de Pós-Graduação em Estudos da Tradução (POSTRAD) \\ germanahp@gmail.com
}

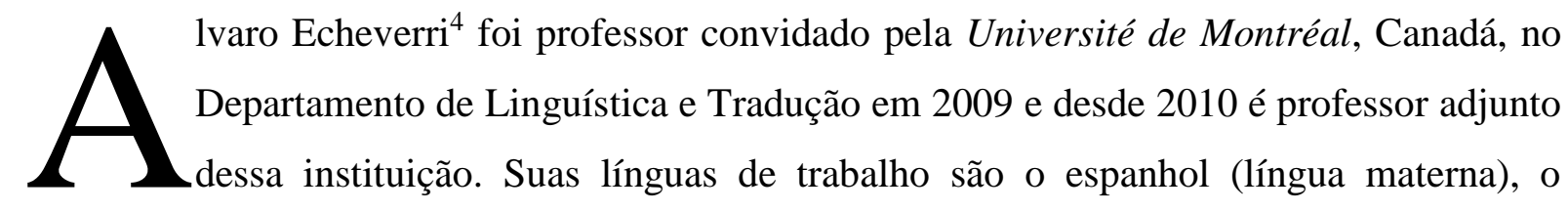

francês e o inglês. Possui graduação em línguas estrangeiras pela Universidad de Antioquia, Medellín, Colômbia (1994), mestrado em Línguas Estrangeiras (M.A in Foreign Languages, West Virginia University, Morgantown (WV), EUA, 2000), mestrado em Tradução (M.A in translation, Kent State University, Kent (OH), EUA, 2001) e doutorado em Traduction, opção Tradutologia pela Université de Montréal, Montréal, Canadá, com tese intitulada "Métacognition, apprentissage actif et traduction : l'apprenant de traduction, agent de sa propre formation" (2008), a qual originou um livro de mesmo título em $2010^{5}$. Sua principal área de interesse está relacionada à pedagogia da tradução, ao papel ativo dos aprendizes em sua formação, em especial à metacognição no processo de ensino-aprendizagem de tradução e sua influência na aplicação de métodos de aprendizagem nos cursos de Tradução. Professor Echeverri se interessa também pela História da Tradução e é membro do Grupo de Pesquisa sobre a História da Tradução na América Latina (HISTAL) http://www.histal.ca/pt, coordenado pelo Professor Dr. Georges Bastin. Além disso, pesquisa acerca do desenvolvimento da tradutologia por meio da análise de traduções de textos sobre a tradutologia em inglês, espanhol e francês após a década de 1970. 
1. O que levou o senhor a se interessar pela tradução? Poderia nos contar um pouco da sua trajetória?

ECHEVERRI: Mi interés por la traducción fue una consecuencia normal del amor que desarrollé por las lenguas extranjeras durante mis estudios de bachillerato. Durante los años que pasé en el liceo desarrollé un gusto por los idiomas. En el colegio siempre obtuve mejores resultados en materias relacionadas con las ciencias sociales y humanas que en materias como algebra, cálculo, trigonometría o física. En particular, el español y las lenguas extranjeras me resultaban mucho más interesantes. La elección de la carrera universitaria fue entonces una consecuencia normal del gusto por los idiomas.

Yo hice mis estudios de pregrado en Lenguas modernas en la Universidad de Antioquia, en Medellín (Colombia). La carrera comprendía una serie de cursos de traducción y ese fue mi verdadero primer contacto con una actividad que he practicado desde mi época de alumno de pregrado. En Colombia, trabajé como intérprete y traductor independiente durante varios años. Luego viajé a Francia (Estrasburgo) con el fin de estudiar traducción.

150 Asistí a varios cursos pero me desmotivó mucho la traducción universitaria que se enseñaba en la universidad francesa a la que yo asistía. Los cursos estaban dirigidos a futuros profesores de lenguas. La traducción era una manera de mejorar los conocimientos lingüísticos de los futuros profesores de español y eso no me interesaba. Esta experiencia en Francia hizo que perdiera un poco de pasión por la traducción y decidí hacer estudios de lingüística.

Cuando regresé a Colombia me inscribí en una especialización en didáctica de lenguas extranjeras. La calidad de los profesores y del programa en general me ayudaron a desarrollar el gusto por la investigación. Sin embargo, tuve la posibilidad de viajar a hacer estudios de postgrado a Estados Unidos y no lo pensé dos veces. Dejé mis estudios de especialización en didáctica de lenguas extranjeras y desde 1998 hasta 2000 hice una maestría en Literatura francesa y TESOL en West Virginia University. En este período hice varios trabajos de traducción y esto me ayudó a recuperar el gusto por la traducción.

Entonces decidí inscribirme en la maestría en traducción (francés-inglés) en Kent State University. El programa de formación en Kent fue tal vez uno de los aspectos que más me motivó a interesarme por todo lo referente a la formación de traductores. Este programa combinaba acertadamente los aspectos teóricos y los aspectos profesionales de la traducción. Aunque el programa tenía un componente vocacional/profesional fuerte, los aspectos teóricos 
eran abordados de tal manera que los alumnos desarrollábamos una idea propia de la traducción. En Kent State University, los alumnos de traducción teníamos la oportunidad de aprender teoría al lado de traductólogos reconocidos como Gregory Shreve, Carol Maier o Françoise Massardier-Kenney, pero siempre con la vista puesta en la práctica. En Kent, la traductología y la traducción literaria y no literaria eran una sola. En otras palabras, en Kent State University, yo aprendí a ser un traductor que reflexiona sobre su práctica. Fue el ambiente educativo de Kent y la idea de una formación integral y pertinente ofrecida en un medio natural lo que me llevó a interesarme por la formación de traductores como campo de estudio. Mi recorrido es entonces el de un traductor que reflexiona sobre su práctica, que no deja de ejercerla pero que la combina con la enseñanza. Yo me considero un traductor que adora enseñar su profesión.

2. O senhor trabalhou como intérprete em hospitais nos Estados Unidos. Como surgiu essa oportunidade e como o senhor vivenciou essa experiência?

ECHEVERRI: La oportunidad de trabajar como interprete en los servicios de salud en el área de Canton (Ohio) surgió gracias a una colaboración entre la Universidad y los agricultores de la región. Los agricultores recibían todos los años una gran cantidad de trabajadores temporales que llegaban principalmente de México y de otros países de Centro América. Los agricultores brindaban un espacio físico, una clínica, en la que se ofrecía atención médica a los trabajadores inmigrantes. La universidad contribuía con los profesionales de la salud. La clínica era un lugar de práctica para los alumnos de medicina que trabajaban al lado de un profesor. Mi trabajo consistía en facilitar la comunicación entre los pacientes y los practicantes o entre los pacientes y el profesor de medicina. La interpretación se hacía en ambas direcciones español-ingleslinglés-español. El trabajo de interpretación en sí mismo no era complicado ya que los intercambios entre el personal médico y los pacientes eran relativamente simples. Lo más duro de este trabajo era tener que vivir muy de cerca la problemática social y humana de los trabajadores agrícolas inmigrantes en Estados Unidos. Este trabajo lo hice durante 10 meses y me dejo experiencias de vida muy importantes.

3. Em 2008, o senhor defendeu a tese intitulada "Métacognition, apprentissage actif et traduction : l'apprenant de traduction, agent de sa propre formation” pela Université de Montréal. As pesquisas relacionadas à formação do tradutor geralmente apresentam o 
conceito de cognição, mas somente a partir da sua tese o conceito de metacognição foi inserido nessa linha de pesquisa. O senhor poderia explicar como esse conceito da psicologia cognitiva pode ser inserido no processo de ensino-aprendizagem nos cursos de tradução?

ECHEVERRI: Un aspecto que se debe tener muy en cuenta cuando se habla de metacognición en la educación en general es que la metacognición no se enseña de manera explicita. Como lo he dicho ya en varias ocasiones, el profesor que pretenda integrar la metacognición en su enseñanza no puede llegar al aula de clase y decir: "Hoy les voy a enseñar la metacognición". La metacognición es un constructo psicológico utilizado para referirse a los procesos cognitivos que permiten al ser humano comprender y controlar su actividad cognitiva. Es decir la metacognición permite a los alumnos conocer mejor su propia capacidad a procesar la información y por consiguiente a controlar mejor sus actividades mentales y el aprendizaje.

Lo más importante es hacer que los alumnos conozcan el concepto. Esto se puede hacer aprovechando el momento de hacer comentarios generales sobre la corrección de un 152 ejercicio de traducción. Por ejemplo, la mayor parte de los problemas que encontramos en los trabajos de los alumnos son problemas de lengua y problemas de conocimientos del tema que se traduce. En general, los problemas de lengua son problemas que los alumnos pueden resolver por sí mismos. Lo que quiere decir que al momento de hacer la traducción o de revisarla, los alumnos no controlaron bien el proceso de lectura (un proceso cognitivo), no se concentraron lo suficiente (otro proceso cognitivo). En el caso de los problemas de conocimientos temáticos, el primer paso es comprender el texto de partida. Antes de traducir se debe estar seguro de haber comprendido adecuadamente el contenido del texto que se traduce. Aunque parezca extraño, siempre hay casos de alumnos que traducen sin estar seguros de haber comprendido el texto de partida. La metacognición es justamente nuestra capacidad a evaluar, a monitorear y a controlar la calidad de nuestra actividad cognitiva. En el aula de clase, existen momentos que se prestan para hablar de metacognición. Al momento de proponer una actividad a los alumnos se puede discutir con ellos sobre los conocimientos que poseen del tema que se va a traducir. Esta es una buena ocasión para permitir que los alumnos hagan una autoevaluación de sus propios conocimientos. Para que se pregunten si sus conocimientos son suficientes antes de empezar a traducir y para que conciban las estrategias que les van a permitir compensar la falta de conocimiento, o que por el contrario van a confirmar que sus conocimientos sobre el tema son realmente suficientes. El principio 
de traducción que hay detrás de esta idea es que el conocimiento de palabras y de términos no es suficiente para traducir. Es necesario manejar, en el sentido de controlar, los conceptos para poder actuar con autonomía y conocimiento sobre los textos y sobre el mensaje.

4. Com quais abordagens propostas para o ensino de tradução a dimensão metacognitiva da tradução se relaciona melhor? (Abordagem funcionalista, Christiane Nord; Abordagem socioconstrutivista, Don Kiraly; Abordagem por objetivos, Jean Delisle; Abordagem por tarefas, Amparo Hurtado Albir; Abordagem situacional, Vienne e Gouadec; Abordagem orientada ao processo, Daniel Gile; Análise consciente e descoberta subliminal, Douglas Robinson.)

ECHEVERRI: La metacognición constituye uno de los pilares teóricos sobre los cuales se empezó a estructurar la formación centrada en el alumno en los años 1970. Además de la metacognición: los procesos cognitivos (conceptualización, comprensión, resolución de problemas, toma de decisiones), las habilidades intelectuales centrales (representación, síntesis, elaboración), el pensamiento crítico y el pensamiento creativo fueron conceptos que los expertos en educación identificaron como los más adecuados para permitir que los sistemas educativos en general se adaptarán al paso de una sociedad manufacturera a una sociedad de servicios y de saber. Es un hecho que el conocimiento avanza cada vez más rápido. Esto explica que, en gran parte, los esfuerzos educativos vayan dirigidos a desarrollar en los alumnos la capacidad de aprender a aprender y aprender para la vida. Es decir, los alumnos además de integrar los conocimientos temáticos deberán estar capacitados para enfrentar el hecho que esos conocimientos evolucionarán cada vez más rápido. En este sentido, la metacognición es mucho más compatible con el enfoque socioconstructivista de la formación de traductores ya que uno de los conceptos claves de la propuesta pedagógica de Kiraly es favorecer la creación de la imagen de sí mismo del futuro traductor. De todos los enfoques propuestos para la formación de traductores ninguno ha dado tanta importancia a los alumnos como agentes activos de la formación como el enfoque socio-constructivo. La metacognición en ese sentido puede ser una herramienta sumamente útil para favorecer la creación de la imagen de sí mismo del traductor. 
5. Atualmente um dos principais nomes relacionados ao ensino de tradução é a professora da Universitat Autònoma de Barcelona, Amparo Hurtado Albir. O senhor vê alguma relação entre o conceito de metacognição e a abordagem proposta por essa professora?

ECHEVERRI: Es cierto que Amparo Hurtado Albir ha hecho una contribución enorme no solo a la formación de traductores sino a la traductología en general. También es cierto que gracias a sus investigaciones y a las investigaciones de los miembros de su grupo de investigación sobre el Proceso de Adquisición de la Competencia Traductora y Evaluación (PACTE) la discusión en torno al concepto de competencia en traducción dio un salto cualitativo extraordinario. En mi opinión, aparte de proponer una idea integral de lo que es la competencia traductora, los trabajos de Amparo Hurtado y su grupo de investigación, cambiaron el horizonte de la investigación en pedagogía y en didáctica de la traducción ya que se distanciaron de discusiones de tipo hermenéutico para fundar sus propuestas en datos concretos obtenidos gracias a la puesta en práctica de metodologías de investigación empírica. Estamos muy habituados a citar los resultados de las investigaciones de PACTE, pero fuera

154 de España, nos hemos interesado muy poco en las estrategias y técnicas de investigación que permitieron al grupo PACTE brindarnos una imagen mucho más clara de la naturaleza de la competencia traductora. Es gracias al esfuerzo de los colegas españoles asociados a PACTE y al grupo AVANTI de la Universidad de Granada que hoy en día la discusión sobre la formación de traductores dejó de ser una práctica meramente especulativa. El discurso sobre la formación de traductores se fundamenta cada vez en conocimientos compartidos y no en opiniones personales expresadas por personas con alguna autoridad en la materia.

En cuanto a la relación entre el concepto de metacognición y los trabajos del grupo PACTE, yo creo que la metacognición entra en la sub-competencia psicofisiológica tal que definida en el modelo holístico y dinámico definido por PACTE. Se puede decir que en cierta manera mi trabajo sobre la metacognición busca el estudio y la valoración de uno entre muchos conceptos que PACTE ubica en esta sub-competencia. Lo que me parece completamente justificable y acertado.

\section{Qual o seu conceito de competência tradutória?}

ECHEVERRI: Hay varias maneras de concebir el concepto de competencia traductora. Los trabajos del grupo PACTE nos ofrecen una idea muy clara de los conocimientos que una 
persona debe adquirir para trabajar como traductor. Así, desde el punto de vista de la formación la competencia traductora es la adquisición de los conocimientos y habilidades que requiere el trabajo del traductor. Ahora bien, una vez terminados los estudios la competencia traductora se convierte en la capacidad de movilizar estos conocimientos y de utilizarlos de manera adecuada al momento de hacer una traducción en un contexto profesional, por ejemplo. Mi concepto de competencia traductora es la capacidad del traductor de movilizar todos sus conocimientos de manera adecuada en el momento en que estos son requeridos.

7. O que diferencia a competência tradutória da competência do tradutor e como a competência metacognitiva relaciona-se com estas?

ECHEVERRI: Como lo explica Kiraly en su libro A Social Constructivist Approach to Translator Education: Empowerment from Theory to Practice, de 2000, la competencia del traductor implica mucho más que la capacidad de producir un texto meta aceptable. La competencia del traductor implica la capacidad de integrarse adecuadamente a la comunidad de practicantes de la traducción. La competencia del traductor implica que este conoce las prácticas profesionales, las normas, las convenciones que rigen el ejercicio de la traducción en cada lugar de trabajo. La competencia del traductor es además la capacidad de trabajar de manera colaborativa con otros agentes implicados en el mundo de la traducción. En otras palabras, un traductor que conoce muy bien sus dos lenguas de trabajo y las culturas que su trabajo pone en contacto podrá tener una competencia traductora muy elevada. Pero si este mismo traductor, por ejemplo, entrega sus proyectos de traducción en un formato distinto al que se le pidió; si no entrega sus trabajos de traducción a tiempo, este traductor no tendrá la competencia del traductor exigida por el medio.

La metacognición está asociada a los dos conceptos, ya que la competencia traductora exige que el futuro traductor conozca las exigencias de su profesión en términos de conocimientos. La metacognición le permitirá por ejemplo determinar que aspectos de una lengua o de una especialidad requieren un mayor esfuerzo. La metacognición es igualmente esencial en la competencia del traductor porque el traductor profesional debe aprender a ejecutar varios proyectos al mismo tiempo. La gestión de los recursos y del tiempo implican que el traductor se conoce a si mismo y puede determinar en que momento la fatiga y el estrés pueden tener una influencia negativa en su desempeño profesional. Determinar sus propios límites es una capacidad metacognitiva. 


\section{Qual a importância da autoavaliação na formação de tradutores?}

ECHEVERRI: Muchas gracias por hacer esta pregunta. Tradicionalmente la evaluación ha sido concebida como una actividad reservada a los profesores. Los instructores a menudo desaprovechamos el potencial de las evaluaciones para afianzar o para resolver dificultades de aprendizaje. La evaluación no debería ser vista únicamente como un tipo de sanción. La evaluación constituye una oportunidad para establecer un diálogo con el alumno. Este diálogo que se establece en forma de retroacción representa, a mi modo de ver, un momento clave de la formación. Desafortunadamente en la formación de traductores hemos dedicado muy pocos esfuerzos al desarrollo de estrategias y de técnicas de retroacción eficaces en términos de resultados y de utilización recursos humanos. Es bien sabido que la evaluación consume gran parte del trabajo de los profesores. También es sabido que ese trabajo no tiene siempre el efecto deseado en el desempeño de los alumnos. Muchos alumnos siguen cometiendo los mismos errores una y otra vez a pesar de la evaluación.

Es por eso que es de vital importancia ayudar a los alumnos a desarrollar estrategias de 156 autoevaluación. Pero el tipo de autoevaluación al que yo me refiero no es ni esporádico ni implica siempre la atribución de una nota. El tipo de autoevaluación que, en mi opinión, se debe promover es de carácter permanente y auto-regulado por el alumno. Sí, el alumno debe aprender a revisar sus traducciones, sus trabajos escritos, pero también debe que aprender a evaluar su comprensión de un concepto, de un proceso, de una idea. Sería muy bueno que encontráramos la manera de ayudar a los alumnos a verificar que comprenden realmente lo que se espera que ellos aprendan. En muchas ocasiones, nos damos cuenta que los alumnos nos dicen que sí entienden, y lo más interesante, es que ellos están convencidos de haber entendido. Sin embargo, si les pedimos que nos expliquen, eso que supuestamente deberían aprender, nos damos cuenta que las cosas no son tan positivas como ellos piensan.

En el caso de los alumnos de traducción, es evidente que no se han desarrollado criterios claros que faciliten las actividades de auto-evaluación. Así por ejemplo, muchos alumnos piensan que un buen traductor es alguien que traduce rápido. En un contexto de formación, la rapidez de la ejecución no debería preocupar a los alumnos. La velocidad en la ejecución de una tarea dependerá siempre del conocimiento y de las habilidades que se poseen para su ejecución. En este sentido, los alumnos deben, para decirlo de otra manera, "aprender a caminar, antes de correr". 
9. Qual a importância do estágio na formação de tradutores tendo como perspectiva a metacognição?

ECHEVERRI: La pasantía (stage en francés) es un momento clave en la formación de los traductores. En primer lugar, el alumno tiene la posibilidad de entrar en contacto directo con la industria. Para muchos alumnos, la pasantía es la primera oportunidad de ver a un traductor en su lugar de trabajo. En segundo lugar, la pasantía permite a los alumnos hacerse una idea de lo que es la industria de la traducción. El alumno conocerá las prácticas utilizadas en la industria para, por ejemplo, administrar todos los documentos y proyectos que marcan la vida de una empresa de traducción. En cuanto a la metacognición, los alumnos podrán medir su estado de preparación con las exigencias propias de la profesión. Este es otro tipo de autoevaluación. La pasantía también le permite al alumno conocer sus ritmos de trabajo. Esta capacidad de gestión es de naturaleza metacognitiva y es muy importante en el contexto de las pasantías. Al tener que trabajar sobre varios proyectos al mismo tempo, el alumno aprender a administrar mejor su tempo y sus esfuerzos. La autogestión, la autoevaluación son dos capacidades metacognitivas que se pueden desarrollar en el contexto de las pasantías. La dimensión metacognitiva de la traducción está sumamente presente durante todo el periodo de duración de la pasantía porque el alumno se distancia del contexto de enseñanza y se ve obligado a funcionar de manera autónoma.

10. Como pode se dar a integração da Ciência da Educação com a Tradutologia de modo a beneficiar as futuras pesquisas referentes ao ensino de tradução?

ECHEVERRI: El vínculo entre las ciencias de la educación y la traductología se hace muy evidente en el aula de clase y en cualquier otra situación cuyo objetivo sea la formación de los traductores. La enseñanza de la traducción se ha concentrado casi exclusivamente en los conocimientos que deben adquirir los futuros traductores y se ha preocupado menos por la forma en que se lleva a cabo esa formación en el aula de clase. La integración de las dos disciplinas se puede dar reconociendo que la formación de traductores implica mucho más que identificar las dificultades que encierra la traducción de una lengua a otra. En la formación de traductores, como en la de cualquier otra disciplina, se debe tener en cuenta que lo que puede ser difícil para una persona puede no serlo para otra. 
Para ser más preciso, las ciencias de la educación ofrecen a la traducción conocimientos sobre el aprendizaje y sobre la enseñanza. El formador de traductores debe ser consciente de los principios pedagógicos sobre los que se apoya su práctica docente. Las ciencias de la educación ofrecen una gran variedad de fórmulas pedagógicas que los profesores de traducción pueden conocer y escoger según el tipo de curso que enseñan. Así, ciertos cursos funcionan mejor cuando se utiliza el aprendizaje por proyectos. Otros cursos se hacen más interesantes cuando se enseñan según el estudio de casos o el taller de lectura.

Por otro lado, el conocimiento que se tiene en las ciencias de la educación de conceptos como la motivación, las estrategias de enseñanza, las estrategias de aprendizaje, o simplemente del aprendizaje, está mucho más avanzado que en la traductología. Sería entonces triste que en la formación de traductores se desconozca esta realidad. Finalmente, si consideramos que la formación de traductores es un campo de investigación en sí mismo entonces se debería mirar a las ciencias de la educación para adaptar sus métodos de investigación.

158 11. A perspectiva ecológica já é bastante utilizada no ensino de línguas estrangeiras. Como os Estudos da Tradução, em especial o ensino de tradução, podem ser beneficiados por esta perspectiva?

ECHEVERRI: Un aspecto que es necesario discutir primero que todo es el hecho de que el aprendizaje no se restringe al aula de clase y que el profesor no es la única fuente de información con la que cuentan los alumnos. Como lo demuestra la bibliografía relativa al enfoque ecológico, el aprendizaje es una realidad compleja que no se explica por la simple relación entre una persona que quiere aprender y una persona que puede enseñar. El enfoque ecológico se funda en la idea que el macro-contexto, todo lo que sucede fuera del aula de clase, desempeña una función esencial en el aprendizaje. Como se observa en la actualidad en Canadá y en Estados Unidos, solo para dar un ejemplo, la mayoría de los alumnos tienen que trabajar para pagar sus estudios. El tiempo que dedican a sus cursos no es el ideal. Las prácticas docentes no pueden seguir ignorando esta realidad. Otro asunto interesante es el hecho que el número de alumnos por clase no deja de aumentar. La formación de traductores requiere una práctica intensiva y solo en la lógica de los administradores se puede pensar que se pueden formar traductores competentes haciendo uno o dos ejercicios prácticos en un periodo de cuatro meses. Con clases de más de 40 alumnos, el profesor que enseña un curso 
práctico de traducción dedicaría gran parte de su tiempo a la revisión del trabajo de los alumnos. Con clases tan grandes es ilógico pensar que se puede dar una retroacción detallada a cada alumno.

Personalmente, yo creo que el enfoque ecológico se puede aplicar con algún grado de éxito en la formación de traductores pero primero se tienen que explorar temas como el aprendizaje autónomo, las estrategias de aprendizaje y otros temas relacionados que deberán ser explorados por los responsables de la formación de traductores. Sin embargo, para no cometer los mismos errores del pasado será necesario preparar el terreno formando a los profesores y sobre todo informando a los alumnos.

\section{Qual seu principal interesse em relação à História da Tradução na América Latina?}

ECHEVERRI: Mi principal interés en la historia de la traducción en América Latina son los textos de carácter político que se tradujeron en la época de la independencia. El análisis de estas traducciones demuestra que la traducción fue un instrumento de guerra adicional en manos de los revolucionarios independentistas. Cuando se considera que los textos políticos fundacionales como las constituciones y las declaraciones de independencia no existían en la región en la época se comprende mejor el papel desempeñado por la traducción en este período. La traducción permitió que en poco tiempo las poblaciones que buscaban la independencia tuvieran un texto que rigiera su vida como repúblicas independientes. Sin la posibilidad de traducir las constituciones francesa y estadounidense, por ejemplo, la redacción de esos documentos hubiera tomado demasiado tiempo. El papel de la traducción en la época de independencia de las repúblicas suramericanas fue esencial en la consolidación del movimiento independentista. Mi interés es pues estudiar el uso que los revolucionarios de la época hicieron de la traducción para lograr la independencia.

13. Como se deu seu interesse pela pesquisa em História da Tradução por meio da análise de textos relacionados à tradutologia?

ECHEVERRI: Mi interés por la traductología traducida surgió en el contexto de las aulas de clase como alumno. En los cursos de teoría de la traducción debíamos leer y comentar textos claves de la traductología. El hecho de que la mayoría de la bibliografía de la disciplina esté escrita en inglés hace que al momento de comentar los textos en una lengua distinta al inglés 
surjan problemas terminológicos y que la comprensión de algunos conceptos no sea, en muchos casos, la más adecuada.

Otro aspecto que despertó mi interés por el tema fue constatar que la traducción, como lo explicaba André Lefevere, es manipulación. Si la traducción ha sido utilizada para dar cierta dirección a la literatura de un país, o de una disciplina, es posible que la traducción de textos traductológicos tenga una influencia en la dirección que ha tomado la traductología. En el caso de la traductología, es evidente que la presencia del pensamiento alemán en la bibliografía traductológica de los años 70 y 80 tuvo mucho que ver con los teóricos de la traducción que decidieron traducir los textos de Goethe, de Walter Benjamin, de Holderlin, entre otros pensadores alemanes. En mi primer viaje a Brasil, en 2014, me sorprendió positivamente ver la cantidad de textos traductológicos que han sido traducidos al portugués. Sería interesante hacer un inventario de esas obras traductológicas traducidas en Brasil para saber si esta actividad traductora tiene alguna influencia en la dirección que toma la disciplina determinará en el país.

160 14. Sabemos que o senhor é "apaixonado" pela sala de aula. Foi esta paixão que o levou a se interessar pela pesquisa acerca do processo formativo do tradutor?

ECHEVERRI: Mi pasión por todo lo que pasa en el aula de clase surgió cuando era alumno de traducción. Yo tuve la suerte de estudiar traducción en Colombia, en Francia, en Estados Unidos y en Canadá. Una de las cosas que me más me sorprendió fue constatar que en todas partes las actividades en el aula de clase eran las mismas en todos los países. La bibliografía traductológica me permitió constatar después que en muchas partes del mundo se repetía la misma situación. Los ejercicios prácticos de traducción se organizan, o se organizaban, de la siguiente manera. En el aula de clase, el profesor entrega un texto que los alumnos deben traducir individualmente. La próxima vez que el profesor y los alumnos se encuentran en el aula de clase, los alumnos cada uno a su turno leen una parte de su traducción y el profesor procede a comentar las propuestas de los alumnos. Como alguien que había hecho estudios en la enseñanza de lenguas extranjeras esta situación me parecía algo extraña. En la enseñanza de lenguas extranjeras yo notaba un poco más de variedad en las actividades de clase. Autores como Jean Delisle, Amparo Hurtado Albir y sobre todo Donald Kiraly fundaron sus propuestas pedagógicas en su rechazo a la falta de innovación y de pedagogía que se refleja en esta manera de enseñar. En mi opinión, los traductólogos españoles llevan una ventaja muy 
importante en este sentido porque desde hace más o menos 20 años han desarrollado actividades de investigación en el aula de clase. Claro que mi pasión por el aula de clase viene también de mi pasión por la enseñanza. Yo creo que no hay lugar más triste en el mundo que una universidad norteamericana en verano cuando todos los alumnos están en vacaciones.

15. Qual seria um currículo mínimo para formação do tradutor em sua opinião? O que falta constar nas grades de cursos dos aprendizes em tradução nas universidades em que o senhor atua?

ECHEVERRI: Yo pienso que en lo referente al currículo la traductología no deja de hacer avances importantes. La organización de los programas de formación responde a necesidades y tradiciones particulares de las sociedades en las que se ofrecen los programas de traducción. Así, en Estados Unidos la formación de traductores tiene lugar sobre todo a nivel de la maestría, la traducción se hace de muchas lenguas hacia el inglés y se acepta fácilmente que muchos traductores traduzcan a su segunda lengua, el inglés. En Canadá, la situación es completamente opuesta. Los traductores se forman a nivel de pregrado, se traduce principalmente entre las dos lenguas oficiales (inglés y francés) y no se ve con muy buenos ojos que los traductores traduzcan a una lengua que no sea su lengua materna. Tanto en Estados Unidos como en Canadá se parte del principio que los alumnos tienen un conocimiento refinado de las lenguas de trabajo al momento de empezar a estudiar. En otros países, la situación es completamente diferente. Las preocupaciones curriculares son particulares a cada contexto, lo que funciona en un país a lo mejor no sea lo más indicado en otro. También hay que tener en cuenta que ha medida que las sociedades cambian la traducción se adapta y por eso los programas de formación debería ser lo suficientemente flexibles para eliminar o para crear cursos ha medida que la situación lo requiera.

\section{PUBLICAÇÕES RELEVANTES}

ECHEVERRI, Álvaro. «Le discours sur la formation des traducteurs : au-delà des questions linguistiques ou la quête de pertinence ». In : BELLE, Marie-Alice; ECHEVERRI, Álvaro (dir). Pour une interdisciplinarité réciproque : Modes et pratiques de la recherche en traductologie.Presses Universitaires d'Artois, Lille. no prelo.

Translator education and metacognition: towards student-centered approaches to translator education. In: CUI, Ying; ZHAO, Wei (dirs). Teaching Language Translation and Interpretation: Methods, Theories, and Trends. IGI Global, 2014, p. 297 - 323. 
- "La traducción española de las obras de Thomas Paine en América: El caso de Sentido común”. In: LAFARGA, Francisco; PEGENAUTE, Luis (dirs) Lengua, cultura y política en la historia de la traducción en Hispanoamérica. Vigo (Espagne), Academia Editorial del Hispanismo, 2012, pp. 53-61

. Métacognition, apprentissage actif et traduction : l'apprenant de traduction, agent de sa propre formation. Saarbrücken : Presses Universitaires Européennes, 2010, 359 p.

BASTIN, Georges. L.; ECHEVERRI, Álvaro; CAMPO, Ángela. "Translation and the Emancipation of Hispanic America". In: TYMOCZKO, Maria (dir). Translation, Resistance, Activism. Amherst and Boston: University of Massachusetts Press, 2010, pp. 42-64

\footnotetext{
${ }^{1}$ Lattes Patrícia Rodrigues Costa. Disponível em: http://lattes.cnpq.br/9546437584230118

${ }^{2}$ Essa entrevista é um dos resultados da estadia em pesquisa na Université de Montréal que me foi concedida pelo governo canadense por meio da bolsa Programme des futurs leaders dans les Amériques (PFLA) sendo o Professor Alvaro Echeverri meu supervisor de pesquisa durante o período de agosto de 2014 a fevereiro de 2015.

${ }^{3}$ Lattes Germana Henriques Pereira de Sousa. Disponível em: http://lattes.cnpq.br/5479032498605468

${ }^{4}$ Alvaro Echeverri. Disponível em: http://ling-trad.umontreal.ca/repertoire-departement/vue/echeverri-ariasalvaro/

${ }^{5}$ COSTA, Patrícia Rodrigues. ECHEVERRI, Alvaro. Métacognition, apprentissage actif et traduction: l'apprenant de traduction, agent de sa propre formation. Berlim: Éditions Universitaires Européenes, 2010, 392p. ISBN13: 9786131529061. Brasília: Revista Belas Infiéis, 2013, v.2, n.2, p. 151 - 158. (Resenha). Disponível em: http://periodicos.unb.br/index.php/belasinfieis/article/view/10628/7694
}

RECEBIDO EM: 05/06/2015

ACEITO EM: 20/06/2015 